\title{
Determination of the critical load of eccentrically compressed columns by the second group of limit states
}

\author{
Evgeniy Galagurya ${ }^{1 *}$, Maksym Kovalov $^{1}$, Larisa Kravtsiv $^{1}$ and Igor Bychenok ${ }^{1}$ \\ ${ }^{1}$ Ukrainian State University of Railway Transport, Structural Mechanics and Hydraulics Department, \\ Feuerbach sq., 7, Kharkiv, 61050, Ukraine
}

\begin{abstract}
Determination of critical load values of eccentrically loaded columns was subject of studies by such authors as Vatulia G. L., Galagurya E. I., Glazunov Y. V., Zhakin I. A., Opanasenko E. V., Storozhenko L. I., Timoshenko S. P., Trull B. A., Hoff N. D., Chikhladze E. D. and other researchers. However, those works have not studied properly work of statically determinable and statically indeterminable columns, compressed eccentrically at one end and being under the axial compression at the other one. The article deals with the problem of determining the critical load for the following design patterns: a column that is loaded at the ends by longitudinal forces by equal and singledirection eccentricities; a column that is rigidly clamped at the bottom, hinged at the top and loaded by longitudinal force at the upper end eccentrically; column rigidly clamped at the bottom, loaded at the free end of the longitudinal force eccentrically; a hinged column, loaded at the upper end of the longitudinal force eccentrically. Critical load of excentrically loaded columns with different types of bearing and load patterns can be determined using the obtained equations. Allowable deflection value according to the regulatory documents and geometric characteristics of the considered columns should be specified to solve these equations.
\end{abstract}

\section{Introduction}

Works by Chikhladze E. [1, 2, 3], Vatulia G. [3], Glazunov Y, Zhakin I., Opanasenko E., Storozhenko L. deal with the experimental and theoretical study of stress-strain state of the centrically and eccentrically compressed columns and other supporting structures. Those works have contributed to solving the difficult problem of assessing the strength and stiffness of columns. However, not all problems have been solved. In particular, action of statically determinate and statically indeterminate columns compressed eccentrically at one end and being under axial compressions at the other has not been studied thoroughly. In this regard, this article provides solutions that allow determining critical load of the following design patterns (Fig. 1).

\footnotetext{
* Corresponding author: galagurya@,rambler.ru
} 


\section{Problem definition}

First the relation between load and deflection which occur in columns under various load patterns should be obtained to determine the critical load of eccentrically compressed columns.

a)

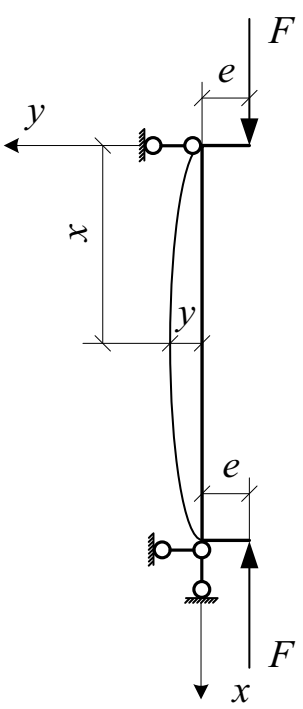

b)

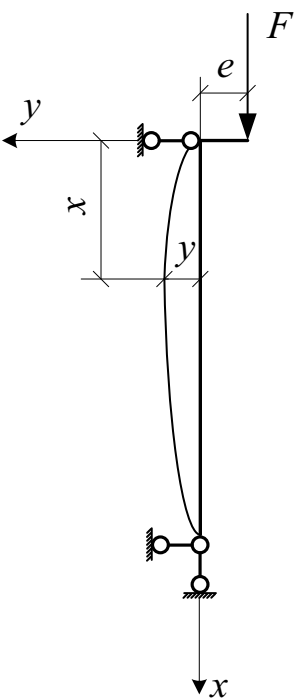

c)

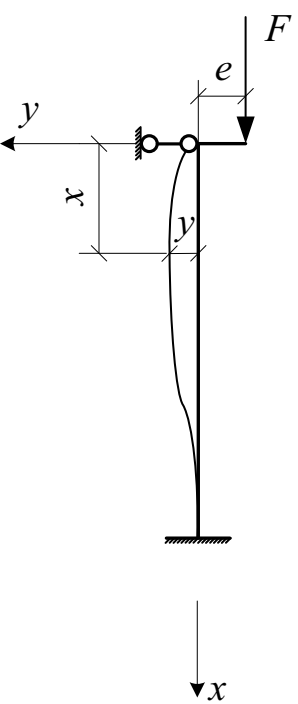

d)

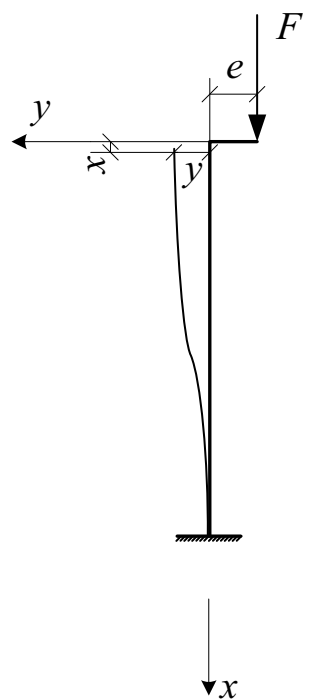

Fig. 1. Design patterns of the columns.

\subsection{Column eccentrically loaded by two compressive forces}

Consider a column loaded by two compressive forces applied with eccentricity $e$ (Fig. 1a). The differential equation of the deflected axis has the form:

$$
y^{\prime \prime}+k^{2} \cdot y=-k^{2} \cdot e
$$

where:

$$
k^{2}=\frac{F}{E I}
$$

solution of equation (1):

$$
y=e\left(\cos k x+\frac{1-\cos k l}{\sin k l} \sin k x-1\right)
$$

The distance between the cross-section with maximum deflection and the origin of coordinates is $x=0.5 l$. The maximum deflection is defined by the formula:

$$
y=e\left(\cos 0.5 k l+\frac{1-\cos k l}{\sin k l} \sin 0.5 k l-1\right)
$$

After we insert the equation (2) into the equation (4), we get the following 


$$
y=e\left(\cos \left(0.5 l \sqrt{\frac{F}{E I}}\right)+\frac{1-\cos \left(l \sqrt{\frac{F}{E I}}\right)}{\sin \left(l \sqrt{\frac{F}{E I}}\right)} \sin \left(0.5 l \sqrt{\frac{F}{E I}}\right)-1\right)
$$

We can express the value of critical force from the equation (5) using the software

$$
F=\frac{4 \operatorname{arctg}\left(\frac{e \cdot \sqrt{2 e y+y^{2}}}{y+e}\right)^{2} E I}{l^{2}}
$$

Substitute the value of the allowable deflection of the column according to $[4,5,6]$ to find the critical force.

\subsection{Hinged column loaded by force $F$ applied eccentrically at the upper end}

Consider a hinged column loaded by eccentrically applied force $\mathrm{F}$ at the upper end (Fig. 1b). The differential equation of the curved axis is:

$$
y^{\prime \prime}+k^{2} \cdot y=\frac{k^{2} \cdot e \cdot x}{l}-k^{2} \cdot e
$$

solution of the equation (7):

$$
y=e\left(\cos k x-\operatorname{ctg} k l \cdot \sin k x-1+\frac{x}{l}\right)
$$

The section which has the maximum deflection will be critical. The point of extreme (8) and the value of $x$ should be found to determine the critical section. Using the software we can find the cross section with the maximum deflection:

$$
x=\frac{2 \operatorname{arctg}\left(\frac{k l \cdot \operatorname{tg} k l-\sqrt{\frac{k^{2} l^{2}-1}{\cos (k l)^{2}}+1}}{k l+\operatorname{tg} k l}\right)}{k}
$$

After we insert the equation (2) and the equation (9) into the equation (8), we get the equation (10). Since the function in the equation (10) is indeterminate, we cannot calculate the value of the critical force analytically, but knowing the geometric characteristics of the column and the allowable deflection, we can get the value of the critical force. 


$$
\begin{aligned}
& y=e \cdot \cos \left(2 \operatorname{arctg}\left(\frac{\sqrt{\frac{F}{E I}} l \cdot \operatorname{tg} \sqrt{\frac{F}{E I}} l-\sqrt{\frac{\frac{F}{E I} l^{2}-1}{\cos \left(\sqrt{\frac{F}{E I}} l\right)^{2}}+1}}{\sqrt{\frac{F}{E I}} l+\operatorname{tg} \sqrt{\frac{F}{E I}} l}\right)-\right. \\
& -e \cdot \operatorname{ctg} \sqrt{\frac{F}{E I}} l \cdot \sin \left(2 \operatorname{arctg}\left(\frac{\sqrt{\frac{F}{E I}} l \cdot \operatorname{tg} \sqrt{\frac{F}{E I}} l-\sqrt{\frac{\frac{F}{E I} l^{2}-1}{\cos \left(\sqrt{\frac{F}{E I}} l\right)^{2}}+1}}{\sqrt{\frac{F}{E I}} l+\operatorname{tg} \sqrt{\frac{F}{E I}} l}\right)-\right.
\end{aligned}
$$

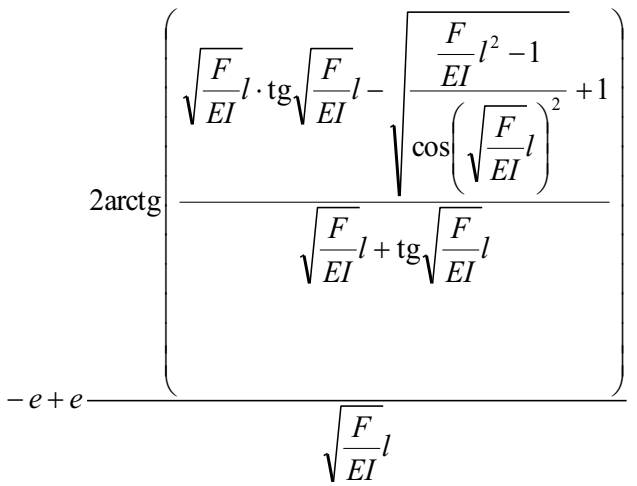

\subsection{Column fixed at the bottom, hinged on top and loaded eccentrically by force applied at the upper end}

Consider a column which is rigidly fixed at the bottom, hinged on top and loaded eccentrically by longitudinal force applied at the upper end (Fig. 1c). The differential equation of the deflected axis will have the following form:

$$
y^{\prime \prime}+k^{2} \cdot y=\frac{k^{2} \cdot e \cdot x}{2 \cdot l}-k^{2}
$$

Solution of the equation (11):

$$
y=e\left(\cos k x-\left(\frac{\cos k l+0.5}{\sin k l}\right) \cdot \sin k x-1+\frac{x}{2 \cdot l}\right)
$$

The point of extreme (12) should be found to determine the critical section. As a result, we will get the distance $x$ to the section with the maximum deflection: 


$$
\begin{aligned}
& x=\frac{1}{k} \cdot 2 \operatorname{arctg}\left(\frac{-4 k l \operatorname{tg}(0.5 k l)}{k l \operatorname{tg}(0.5 k l)^{2}-3 k l-6 \operatorname{tg}(0.5 k l)}\right)+ \\
& +\frac{\sqrt{k^{2} l^{2} \operatorname{tg}(0.5 k l)^{4}+10 k^{2} l^{2} \operatorname{tg}(0.5 k l)^{2}+9 k^{2} l^{2}-36 \operatorname{tg}(0.5 k l)}}{k l \operatorname{tg}(0.5 k l)^{2}-3 k l-6 \operatorname{tg}(0.5 k l)}
\end{aligned}
$$

After we insert the equation (2) and the equation (13) into the equation (12), we get the equation (14), in which the function is indeterminate and we cannot calculate the value of the critical force analytically, but knowing the geometric characteristics of the column and the allowable deflection, we can get the value of the critical force.

$$
\begin{aligned}
& y=e \cos \left(2 \operatorname{arctg}\left(\frac{l \sqrt{\frac{F}{E I}} \operatorname{tg}\left(l \sqrt{\frac{F}{E I}}\right)-\sqrt{\frac{F l^{2}}{E l \cos \left(l \sqrt{\frac{F}{E I}}\right)^{2}}+1-\frac{1}{\cos \left(l \sqrt{\frac{F}{E I}}\right)^{2}}}}{l \sqrt{\frac{F}{E I}}+\operatorname{tg}\left(l \sqrt{\frac{F}{E I}}\right)}\right)-\right. \\
& -e \operatorname{ctg}\left(l \sqrt{\frac{F}{E I}}\right) \sin \left(2 \operatorname{arctg}\left(\frac{l \sqrt{\frac{F}{E I}} \operatorname{tg}\left(l \sqrt{\frac{F}{E I}}\right)-\sqrt{\frac{F l^{2}}{E l \cos \left(l \sqrt{\frac{F}{E I}}\right)^{2}}+1-\frac{1}{\cos \left(l \sqrt{\frac{F}{E I}}\right)^{2}}}}{l \sqrt{\frac{F}{E I}}+\operatorname{tg}\left(l \sqrt{\frac{F}{E I}}\right)}\right)-\right. \\
& \operatorname{larctg}\left(\frac{l \sqrt{\frac{F}{E I}} \operatorname{tg}\left(l \sqrt{\frac{F}{E I}}\right)-\sqrt{\frac{F l^{2}}{E l \cos \left(l \sqrt{\frac{F}{E I}}\right)^{2}}+1-\frac{1}{\cos \left(l \sqrt{\frac{F}{E I}}\right)^{2}}}}{l \sqrt{\frac{F}{E I}}+\operatorname{tg}\left(l \sqrt{\frac{F}{E I}}\right)}\right)
\end{aligned}
$$

\subsection{Column rigidly fixed at the bottom, loaded at the free end by eccentric force}

Consider a column which is rigidly fixed at the bottom, loaded at the free end by eccentric force (Fig. 1d). The differential equation of the deflected axis will have the following form:

$$
y^{\prime \prime}+k^{2} \cdot y=-k^{2} \cdot e
$$

Solution of the equation (15):

$$
y=e\left(\cos k x+\frac{1-\cos k l}{\sin k l} \sin k x-1\right)
$$

After we set the first-order derivative of the function (16) to 0, we will determine the value of $x$ where the function tends to the maximum value (i.e. find the cross-section with the maximum deflection). We use the software for this purpose. 


$$
x=-\frac{\operatorname{arctg}\left(\frac{\cos (0.5 k l)-1}{\sin (0.5 k l)}\right)}{k}
$$

After we insert the equation (2) and the equation (17) into the equation (16), we get the following

$$
y=e\left(\begin{array}{l}
\cos \left(-\operatorname{arctg}\left(\frac{\cos \left(0.5 l \sqrt{\frac{F}{E I}}\right)-1}{\sin \left(0.5 l \sqrt{\frac{F}{E I}}\right)}\right)\right)+ \\
+\frac{1-\cos l \sqrt{\frac{F}{E I}}}{\sin l \sqrt{\frac{F}{E I}}} \sin \left(-\operatorname{arctg}\left(\frac{\cos \left(0.5 l \sqrt{\frac{F}{E I}}\right)-1}{\sin \left(0.5 l \sqrt{\frac{F}{E I}}\right)}\right)\right)-1
\end{array}\right)
$$

We can express the value of critical force from the equation (18) using the software

$$
F=\frac{4 \operatorname{arctg}\left(\frac{2 e \cdot \sqrt{2 e y+y^{2}}}{(y+e)^{2}}\right)^{2} E I}{l^{2}}
$$

The value of the allowable deflection of the column according to $[4,5,6]$ should be used to calculate the critical force.

\section{Experimental studies}

Experimental studies have been carried out using the example of a column loaded by eccentrically applied force F at one end (Fig. 1b) to verify the obtained equations. A steel square electric-welded cold-deformed pipe (Fig. 2) with a length of $l=900 \mathrm{~mm}$ has been used as a column.

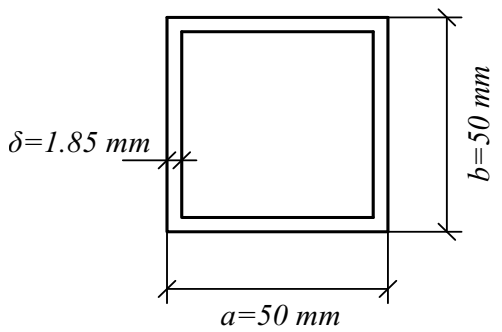

Fig. 2. Cross-sectional diagram of the prototype model.

In order to obtain the data on the physicomechanical characteristics of steel, steel strips have been tested according to [7]. After testing of control samples, the following characteristics have been obtained: yield strength of steel $\sigma_{a}=245 \mathrm{MPa}$, modulus of elasticity of steel $E_{a}=1.27 \cdot 10^{5} \mathrm{MPa}$.

Steel columns have been tested using the hydraulic press PMM125. Bearing assemblies provided a hinged pattern for sample fastening. The load was applied with an eccentricity 
of $25 \mathrm{~mm}$. During the tests, displacement of the sample was measured. Dial gauge indicators IMIG with the division value of $0.01 \mathrm{~mm}$ were used for this purpose. The instruments were installed in the critical section estimated according to formula (9).

Table 1 and Fig. 3 show the values of deflections in critical sections of experimental samples and their comparison with theoretical values, while Table 2 summarizes the values of critical loads of prototypes and their comparison with the theoretical values.

Table 1. Comparison of deflections.

\begin{tabular}{|c|c|c|c|c|c|c|c|c|c|}
\hline \multirow{2}{*}{$\begin{array}{c}F \\
{[\mathrm{kN}]}\end{array}$} & \multicolumn{3}{|c|}{ Sample 1 } & \multicolumn{3}{c|}{ Sample 2 } & \multicolumn{3}{c|}{ Sample 3 } \\
\cline { 2 - 10 } & $\delta_{\exp }$ & $\begin{array}{c}\delta_{\text {theor }} \\
{[\mathrm{mm}]}\end{array}$ & $\begin{array}{c}\Delta \\
{[\%]}\end{array}$ & $\begin{array}{c}\delta_{\exp } \\
{[\mathrm{mm}]}\end{array}$ & $\begin{array}{c}\delta_{\text {theor }} \\
{[\mathrm{mm}]}\end{array}$ & $\begin{array}{c}\Delta \\
{[\%]}\end{array}$ & $\begin{array}{c}\delta_{\exp } \\
{[\mathrm{mm}]}\end{array}$ & $\begin{array}{c}\delta_{\text {theor }} \\
{[\mathrm{mm}]}\end{array}$ & $\begin{array}{c}\Delta \\
{[\%]}\end{array}$ \\
\hline 0 & 0 & 0 & & 0 & 0 & & 0 & 0 & \\
\hline 10 & 0.09 & 0.08 & 11 & 0.098 & 0.08 & 22 & 0.07 & 0.08 & 12 \\
\hline 20 & 0.205 & 0.168 & 22 & 0.172 & 0.168 & 2.3 & 0.17 & 0.168 & 1.1 \\
\hline 30 & 0.289 & 0.266 & 8.6 & 0.243 & 0.266 & 8.6 & 0.253 & 0.266 & 4.8 \\
\hline 40 & 0.4 & 0.375 & 6.6 & 0.393 & 0.375 & 4.8 & 0.372 & 0.375 & 0.8 \\
\hline 50 & 0.5 & 0.499 & 0.2 & 0.497 & 0.499 & 0.4 & 0.498 & 0.499 & 0.2 \\
\hline
\end{tabular}

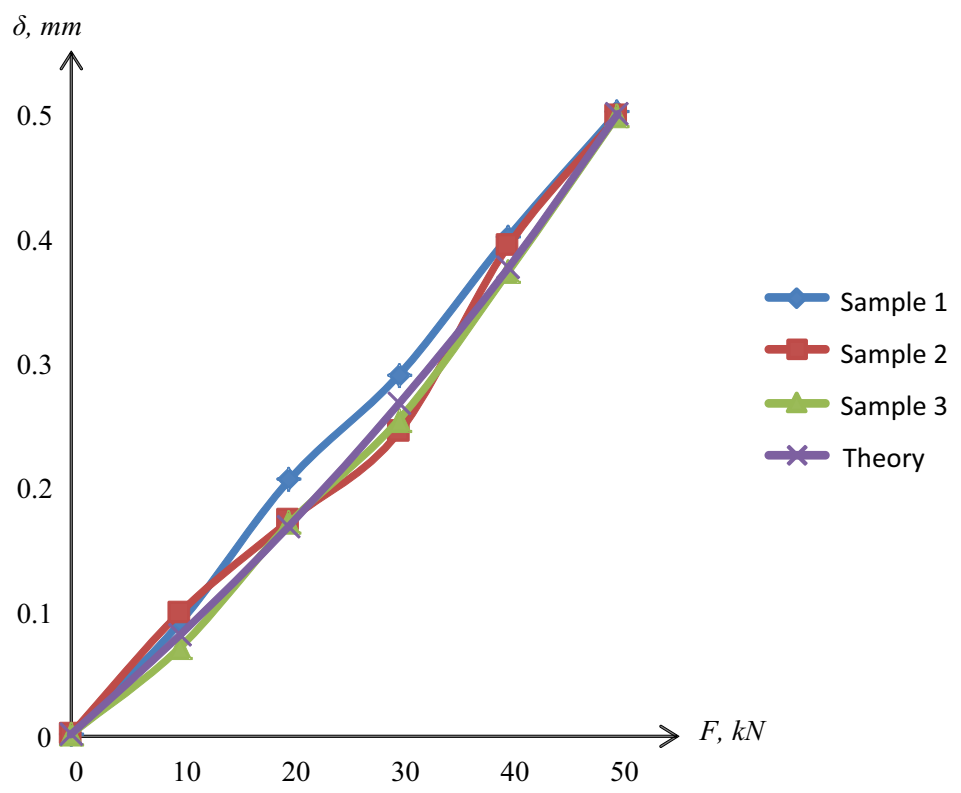

Fig. 3. Comparison of deflections.

Table 2. Comparison of loads.

\begin{tabular}{|c|c|c|c|}
\hline Sample No. & $F_{\exp }[\mathrm{kN}]$ & $F_{\text {theor }}[\mathrm{kN}]$ & $\Delta[\%]$ \\
\hline 1 & 48 & 51.91 & 7.5 \\
\hline 2 & 50 & 51.91 & 3.6 \\
\hline 3 & 49 & 51.91 & 5.6 \\
\hline
\end{tabular}

\section{Conclusions}

Equations to determine the critical load of eccentrically compressed columns have been obtained. Allowable deflection value according to the $[4,5,6]$ and geometric characteristics 
of the considered columns should be specified to solve these equations. These formulas have been confirmed experimentally for a hinged column. As a result of the conducted experimental studies, data on movements in the critical section of experimental columns and their carrying capacity value were obtained.

\section{References}

1. E.D. Chikhladze, Proceedings of Institute of KhIRTE. No. 21 (1993)

2. E.D. Chikhladze, A.D.Arslankhanov, Proceedings of the universities. Building. No. 1 (1992)

3. E.D. Chikhladze, G.L.Vatulya, Kiev, Transport of Ukraine, (2006)

4. DSTU B. V.1.2-3: 2006. Deflections and moving. Requirements engineering. State Standard of Ukraine (2007)

5. DSTU B. V.2.6-75: 2008. Construction of houses and buildings. Construction metal building. General specifications. State Standard of Ukraine (2008)

6. DBN V 2.6-163: 2010. Steel constructions. The rules of designing, manufacturing and installation. State building codes Ukraine. K. Minregionbud Ukraine (2010)

7. DSTU 7305:2013. Metals. Methods of tension test. State Standard of Ukraine (2013) 